\title{
Slant Correction of Vehicle License Plate Image
}

\author{
Lin Liu, Sanyuan Zhang, Yin Zhang, and Xiuzi Ye \\ College of Computer Science / State Key Lab of CAD\&CG, \\ Hangzhou, Zhejiang University, China \\ LiuLinazju.edu.cn
}

\begin{abstract}
Because of perspective distortion between the camera and the license plate, slanted images commonly appear in License Plate Recognition System (LPR system), and it seriously affects the recognition result. This paper presents two types of image slant, and gives an efficient way to rectify them. For horizontal slant correction, the proposed method is based on connected areas labeling and straight-line fitting. For vertical slant correction, the method is based on rotation experiments with various angles. Practical use in the LPR system shows that this method has a correct rate above $97 \%$.
\end{abstract}

\section{Introduction}

\subsection{Vehicle License Plate Recognition System}

Vehicle License Plate Recognition (LPR) is an important part of Intelligent Traffic System (ITS), and it is widely used in traffic control, traffic management and vehicle security. Commonly LPR system includes the following parts: vehicle image acquisition, license plate image location, image pre-processing and character recognition. The flowchart of our LPR system is described in Fig. 1.

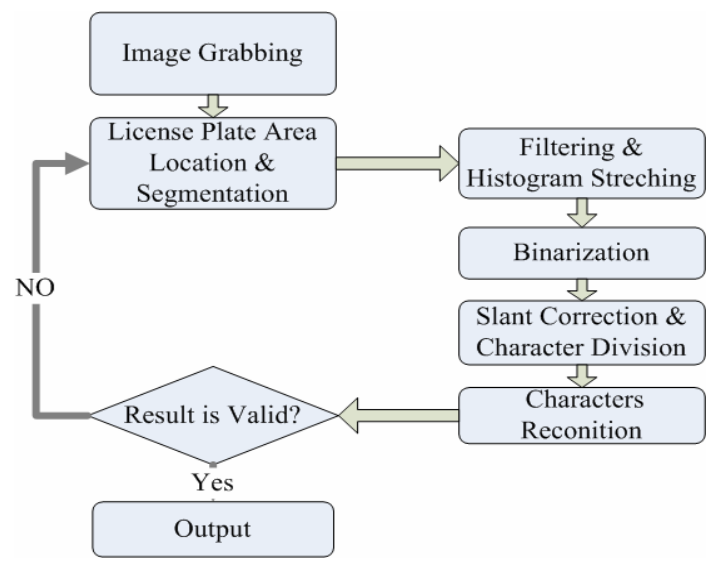

Fig. 1. The flowchart of our License Plate Recognition System 
There is a feedback function in the flowchart. If the recognition result cannot satisfy the syntax requirement (for example, not enough characters), the license plate area is relocated and the license plate is recognized again.

In general, license plate area location decides whether the license plate can be recognized or not, and the image processing module decides final recognition accuracy. Image slant correction is an important part of image processing, which affects the accuracy of character division, even final result.

\subsection{Two Types of Image Slant}

A segmented license pate image is ideally a rectangle aligning with the vehicle image bottom line, but in practice the license plate image is a parallelogram. This leads to the difficulty of character subdivision. There are two types of image slant, namely horizontal slant and vertical slant, as shown in Fig.2 and Fig.3 respectively. Horizontal image slant often leads to integral slant of license plate image, while vertical slant often leads to characters slant. Two real examples of slanted image with their horizontal projection images are given in Fig. 4.

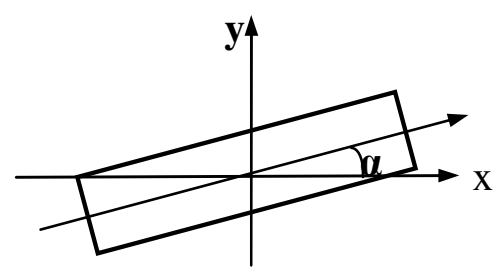

$\alpha>0$

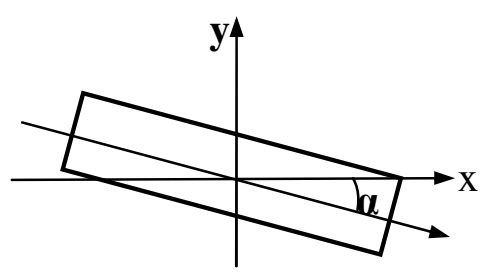

$\alpha<0$

Fig. 2. Two forms of horizontal slant
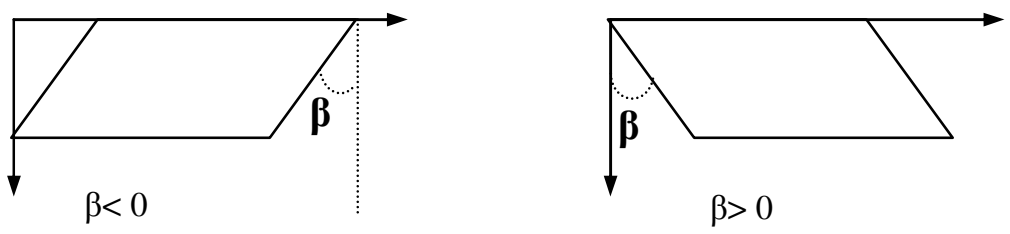

Fig. 3. Two forms of vertical slant

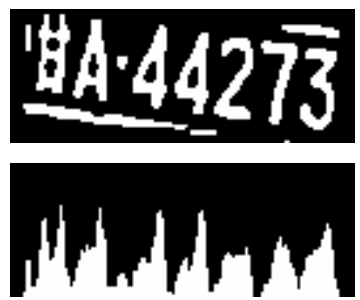

(a) Horizontal slanted image
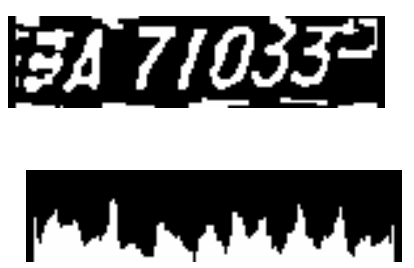

(b) Vertical slanted image

Fig. 4. Two examples of slanted license plate images and their horizontal projections 


\subsection{Previous Work and Our Method}

Slant correction is an important part of LPR system, and it has been intensively studied. A method based on Hough transformation is presented in [1]. It extracts the license plate's top edge and bottom edge using Hough transformation, and the slanted angle is deduced from the edges. A model-matching method is given in [2]. It finds vehicle plate image's four corners by model-matching, then slanted vehicle plate images are transformed to a rectangle area by means of bilinear transformation. A gray value projection method is described in [3][4]. Here the projection image is produced and the license plate area is located by local maximum and minimum projection values. The slant angle is also determined by the projection result. However, it is sometimes difficult to extract the license plate's edges from the background by regular binarization methods (e.g., a white plate on a white vehicle); and due to signal noise and contamination, the plate's edge may be discontinuous; the peak point of Hough transformation is scattered and it is hard to get the acute position of the plate's edge. Therefore, in practice methods based on the plate's edges [1][2] may not get expected results. When the license plate is seriously contaminated, the method in [3] will not work properly.

During our software development, we found that it is relatively easy to separate single character from the whole license plate areas. For horizontal slanted images, if enough character areas are valid, we can use feature points of these character areas to fit a line, and the line can represent the direction of the license plate image. For vertical slanted license plate, its direction is calculated by an empirical method.

\section{Horizontal Slant Correction}

The flowchart of horizontal slant correction is given in Fig.4. We will ignore image segmentation and image binarization steps in this paper, and focus on other four steps in Fig.5.

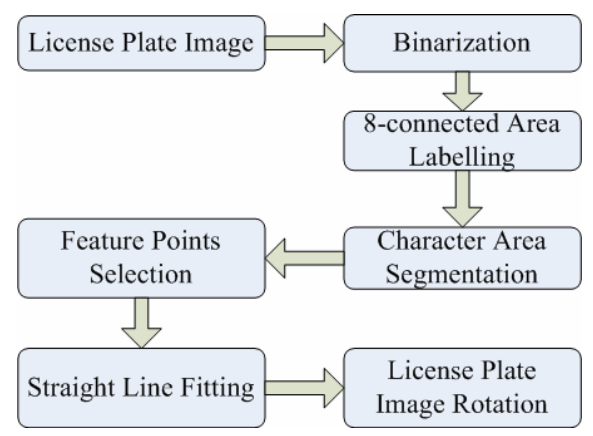

Fig. 5. The flowchart of horizontal slant correction

\subsection{8-Connected Areas Labeling}

The first step of horizontal slant correction is isolating connected areas from a license plate image, which is implemented by connected areas labeling on binaried license 
plate image. After appropriate binarization (here our binarization method is based on OSTU method with a Log filter), an 8-connected area labeling method, rather than a 4-connected one, is adopted to get connected image areas because some image areas are partly discontinuous.

\subsection{Character Areas Selection}

To separate character areas from noise area or license plate's edges, a connected image area with the following characteristics is considered as a valid character area:

(a) The candidate areas are not too wide, and can be separated from the license plate's horizontal edges.

(b) The candidate areas are not too high.

(c) Most candidate areas have a gap among them.

If more than 3 valid character areas are found, it is enough to decide the slant angle of the license plate.

\subsection{Feature Points Selection and Straight-Line Fitting}

Top-most points and bottom-most points of each valid character area are selected as feature points, and they are used to fit the top straight-line and bottom straight-line separately using least square algorithm. The angle between top straight line and bottom straight line is calculated to validate the result, if absolute value of the angle is small ( commonly $<3^{\circ}$ ), then the result is considered valid, otherwise the result is discarded. Fig.6 is an example of the result of straight-line fitting (gray line on the image).

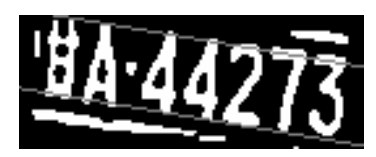

Fig. 6. An example of straight-line fitting

\subsection{Rotation of License Plate Image}

With image rotation, we can get corrected image. Image rotation is not performed on binarized images. This is because direct image rotation on binaried images will bring some noises to the pixels. Fig.7 shows this effect of direct image rotation from Fig.6.

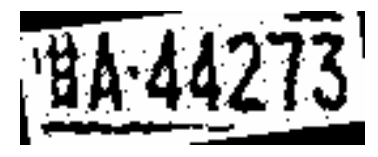

Fig. 7. Direct image rotation's result of Fig.6 
Instead, image rotation is performed on the gray image, and final corrected image is obtained from rotated gray image by a binarization step. Fig. 8 gives the final result.
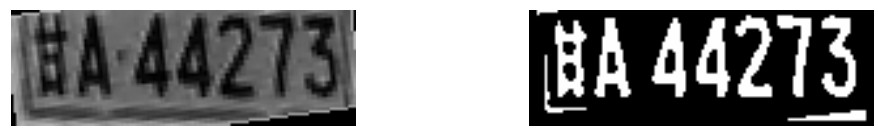

(a) Rotated gray image of Fig.6

(b) Final corrected image (Binarized image of (a))

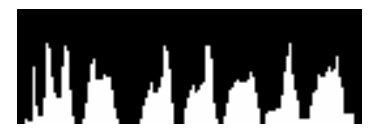

(c) Projection image of (b)

Fig. 8. Image rotation result

Compared with the projection image in Fig.4 (a), we can see that the peak and hollow of Fig.8 (c) is clearer, this will help characters division in Fig.8 (b). In practice, only license plate images slanted with a relatively big angle will be rotated, commonly absolute value of this angle is above $3^{\circ}$.

\section{Vertical Slant Correction}

Vertical slant images are corrected based on the following facts:

(a) 8-connected areas labeling method can isolate one or more character areas.

(b) The horizontal projection width is smallest when the license plate is not slanted.

(c) Slant angle of the license plate is in a specified range, here we give the range from $-20^{\circ}$ to $+20^{\circ}$.

Here we give a simple method to compute the rotation angle: at first a character area is selected, then its horizontal projection is calculated with various angles, finally the angle with smallest projection is selected as the license plate's rotation angle.

For the vertical slanted license plate image given in Fig.4, Table 1 gives the rotation result of selected character.

Table 1. Rotation result of selected character in Fig.4

\begin{tabular}{|l|l|l|l|l|l|l|l|l|}
\hline $\begin{array}{l}\text { Rotation } \\
\text { result }\end{array}$ & $\mathbf{f}$ & $\boldsymbol{f}$ & $\boldsymbol{f}$ & $\boldsymbol{f}$ & $\boldsymbol{f}$ & $\boldsymbol{f}$ & $\boldsymbol{f}$ & $\boldsymbol{\gamma}$ \\
\hline $\begin{array}{l}\text { Rotation } \\
\text { angle }\left(^{\circ}\right)\end{array}$ & -20 & -15 & -5 & 0 & 5 & 10 & 15 & 20 \\
\hline
\end{tabular}

From this table, we can find the rotation angle is $-20^{\circ}$. The result of vertical slant correction of Fig.4 is given in Fig.9. 


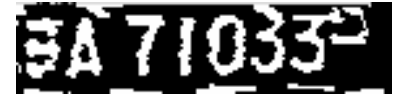

(a) Corrected image

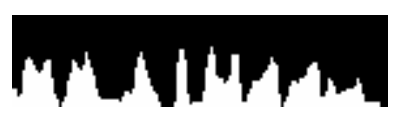

(b) The projection of corrected image

Fig. 9. Result of vertical slant correction

Compared with the projection image in Fig.4 (b), we can see that the peak and hollow of Fig.9 (b) is clearer and easier to distinguish. This will help the subdivision of characters in Fig.9 (a).

\section{Experiment Results and System Performance}

Our method has been embedded into a vehicle license plate system which is very successful commercially. To obtain the performance of this method, we tested more than 1500 license plate images. These plate images are got in various illumination conditions. In Fig.10 and Fig.11, we give more examples of horizontal and vertical slant correction. From Table 2 and Table 3 we can find that there are much more horizontal slanted images than vertical ones. Comparing Table 4 with Table 5 , we can find less license plate images are discarded and the recognition ratio is significantly increased after image slant corrections. The system's recognition ratio is above $88.3 \%$, which can fulfill most regular requirements.

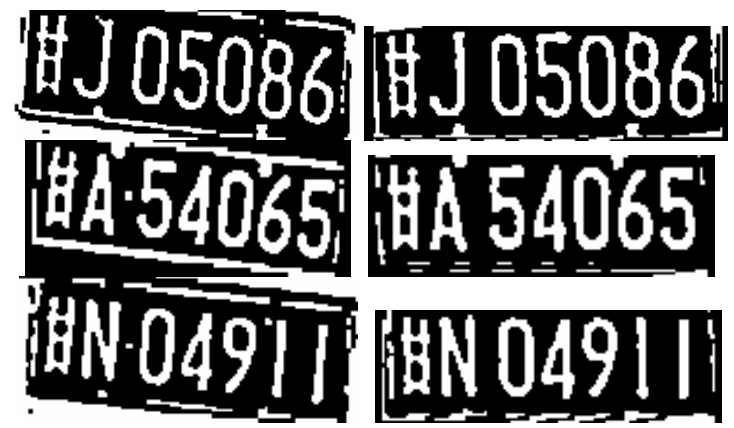

Fig. 10. Examples of horizontal slant corrections

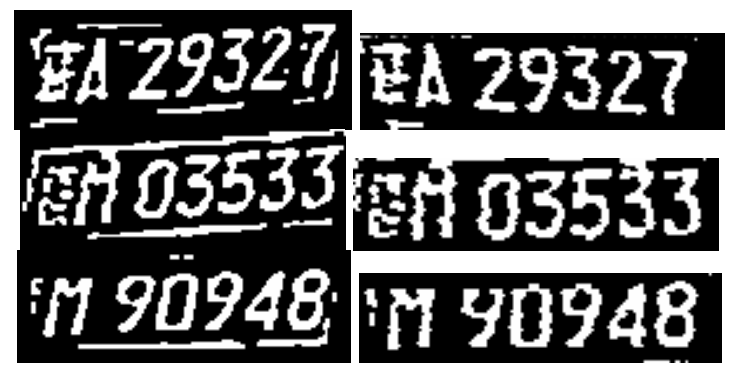

Fig. 11. Examples of vertical slant corrections 
Table 2. Horizontal Slant Correction Ratio

\begin{tabular}{|l|l|l|l|}
\hline $\begin{array}{l}\text { Total license plate } \\
\text { images }\end{array}$ & $\begin{array}{l}\text { Horizontal } \\
\text { slanted images }\end{array}$ & $\begin{array}{l}\text { Corrected } \\
\text { images }\end{array}$ & $\begin{array}{l}\text { Correction } \\
\text { ratio }\end{array}$ \\
\hline 1590 & 688 & 674 & $97.9 \%$ \\
\hline
\end{tabular}

Table 3. Vertical Slant Correction Ratio

\begin{tabular}{|l|l|l|l|}
\hline $\begin{array}{l}\text { Total license plate } \\
\text { images }\end{array}$ & $\begin{array}{l}\text { Vertical slanted } \\
\text { images }\end{array}$ & $\begin{array}{l}\text { Corrected } \\
\text { images }\end{array}$ & $\begin{array}{l}\text { Correction } \\
\text { ratio }\end{array}$ \\
\hline 1590 & 96 & 88 & $91.6 \%$ \\
\hline
\end{tabular}

Table 4. Total Recognition Ratio without Slant Correction

\begin{tabular}{|l|l|l|l|}
\hline $\begin{array}{l}\text { Total license } \\
\text { plate images }\end{array}$ & $\begin{array}{l}\text { Correctly Recognized } \\
\text { images }\end{array}$ & $\begin{array}{l}\text { Discarded } \\
\text { images }\end{array}$ & $\begin{array}{l}\text { Correction } \\
\text { ratio }\end{array}$ \\
\hline 1590 & 1308 & 185 & $82.2 \%$ \\
\hline
\end{tabular}

Table 5. Total Recognition Ratio with Slant Correction

\begin{tabular}{|l|l|l|l|}
\hline $\begin{array}{l}\text { Total license } \\
\text { plate images }\end{array}$ & $\begin{array}{l}\text { Correctly Recognized } \\
\text { images }\end{array}$ & $\begin{array}{l}\text { Discarded } \\
\text { images }\end{array}$ & $\begin{array}{l}\text { Correction } \\
\text { ratio }\end{array}$ \\
\hline 1590 & 1405 & 42 & $88.3 \%$ \\
\hline
\end{tabular}

\section{Conclusion}

Slant correction is an integrant part of vehicle license plate recognition system, and there are commonly two main image slant types: horizontal slant and vertical slant. This paper gives an efficient method to correct slanted images in a commercial license plate recognition system. For horizontal slant correction, our method is based on connected areas labeling and straight-line fitting. For vertical slant correction, our method is based on an empirical method. Practical uses in commercial applications show that our method has a satisfactory correction ratio in all kinds of environmental conditions. Intensive study on the proposed method shows that it is also suitable for slant correction of OCR system.

\section{Acknowledgements}

The authors would like to thank the support from the China NSF under grant \#602720601, China Ministry of Education under grant \# 20030335064, and China Ministry of Science and Technology under grant \#2003AA4Z1020, and the project(No G20030433) of the Education Office of Zhejiang Province 


\section{References}

1. Wen C-Y, Yu C-C, Hun Z-D. A 3-D transformation to improve the legibility of license plate numbers [J], Journal of Foresensic Sciences, 2002, 47(3): 578 585.

2. Hegt H A, Haye R J, Khan N A. A high performance license plate recognition system [A], IEEE international Conference on Systems, Man, and Cybernetics [C]. 1998, 5.4357 4362.

3. van Leeuwen, J. (ed.): Computer Science Today. Recent Trends and Developments. Lecture Notes in Computer Science, Vol. 1000. Springer-Verlag, Berlin Heidelberg New York (1995)

4. Daiyan,Ma Hongqing,,A high performance license plate recognition system based on the web technique, 2001 IEEE Intelligent Transportaion Systems Conference Proceedings (CA)Oakland,USA-August 25-29 